\title{
To Exist and to Count: a Note on the Minimalist View
}

\author{
Francesco Berto and Massimiliano CARrara ${ }^{\dagger}$
}

\begin{abstract}
Sometimes mereologists have problems with counting. We often don't want to count the parts of maximally connected objects as full-fledged objects themselves, and we don't want to count discontinuous objects as parts of further, full-fledged objects. But whatever one takes "fullfledged object" to mean, the axioms and theorems of classical, extensional mereology commit us to the existence both of parts and of wholes - all on a par, included in the domain of quantification - and this makes mereology look counterintuitive to various philosophers.

In recent years, a proposal has been advanced to solve the tension between mereology and familiar ways of counting objects, under the label of Minimalist View. The Minimalist View may be summarized in the slogan: "Count $x$ as an object iff it does not overlap with any $y$ you have already counted as an object". The motto seems prima facie very promising but, we shall argue, when one looks at it more closely, it is not. On the contrary, the Minimalist View involves an ambiguity that can be solved in quite different directions. We argue that one resolution of the ambiguity makes it incompatible with mereology. This way, the Minimalist View can lend no support to mereology at all.

We suggest that the Minimalist View can become compatible with mereology once its ambiguity is solved by interpreting it in what we call an epistemic or conceptual fashion: whereas mereology has full metaphysical import, the Minimalist View may account for our ways of selecting "conceptually salient" entities. But even once it is so disambiguated, it is doubtful that the Minimalist View can help to make mereology more palatable, for it cannot make it any more compatible with commonsensical ways of counting objects.
\end{abstract}

\section{The Minimalist View as a count policy}

Classical, extensional mereology (from now on just "mereology", for short) faces a "tension between countenancing and counting" (Varzi 2000, 284). If a given object $x$ has parts, classical mereology treats the parts as objects in the domain of quantification, on a par with the object $x$ itself. This seems to fall foul of our intuition that many objects have parts that do not count as full-fledged objects themselves, because they are undetached, cognitively irrelevant, and to a large extent arbitrary: the middle third of a baseball bat, for instance, or the southern half of the Empire State Building.

${ }^{\dagger}$ Francesco Berto, Department of Philosophy, University of Venice-Ca'Foscari, Dorsoduro, 3246, 30123 Venice, Italy; E-mail: bertofra@ unive.it. Massimiliano Carrara, Department of Philosophy, University of Padua, P.zza Capitaniato 3, 351339 Padova, Italy; E-mail: massimiliano.carrara@unipd.it

${ }^{1}$ By which we mean: a mereology including (a) some version of the Extensionality Principle, to the effect that for any (non-atomic) $x$ and $y$, if $x$ and $y$ have the same (proper) parts, then $x=y$, and (b) some version of Unrestricted Composition, to the effect that given any $x$ and $y$, there is a (unique) mereological fusion or $\operatorname{sum} z=x+y$. 
On the other hand, given any two objects $x$ and $y$, mereology treats their mereological sum $z=x+y$ as an object in the domain of quantification, on a par with $x$ and $y$ themselves, because of its principle of Unrestricted Composition. This flies in the face of another pervasive intuition, according to which many mereological sums or fusions do not count as full-fledged objects, because they are discontinuous, irrelevant, and to a large extent arbitrary: the fusion of the southern half of the Empire State Building and the middle third of our bat, for instance. As ontologists, we may not want to count undetached parts and disconnected wholes of these kinds. In a word: we need a count policy. Otherwise, mereology is liable of providing an implausible ontology, to say the least (see Chisholm 1987; van Inwagen 2002).

The Minimalist View, proposed by Achille Varzi in order to provide the required count policy, goes as follows:

We should not include [in our inventory of the world] entities that overlap, i.e., share common parts. If we include the table we should not include its top and legs. If we include its top and legs we ought to disregard the whole table as well as every other table part. For instance, we ought to disregard the right half of the table, consisting of the two right legs and the right half of the top. Call this the Minimalist View. The Minimalist View says nothing specific about mereology, about what entities are parts of what. But given a mereological theory and a corresponding domain of quantification, the view tells us how to weigh our ontological commitments:

(M) An inventory of the world is to include an entity $x$ if and only if $x$ does not overlap any other entity $y$ that is itself included in that inventory (Varzi 2000, 285).

This passage includes not only a concise statement of the Minimalist View, but also what we take to be its basic ambiguity - an ambiguity that threatens to make it unsuitable for mereology. In order to disclose the trouble, we will make a couple of assumptions concerning identity.

Assumption (1): it is not the case that identity is only relative to a sortal term. It is not the case, that is, that there is no such thing as identity tout-court, but only non-Leibnizian relations of the form " $x$ is the same $F$ as $y$ ", which do not entail congruence with respect to all properties.

Assumption (2): there is no such thing as worldly vague identity, that is to say: for no $x$ and $y$ can it be indeterminate de re whether $x=y$.

These two assumptions are shared by most mereologists - including Varzi. As for (1), for instance, David Lewis rejects the doctrine of relative identity by deeming it "incredible", and "not [the real] identity, as may be seen from the fact that it sometimes holds between things that differ in their other relative identities" (Lewis 1986, 249). Varzi (2001, 93-94) also appears to follow Lewis's position.

As for (2) - the de re vagueness of identity - "the only intelligible account of vagueness locates it in our thought and language", that is to say, "vagueness is semantic indecision" (Lewis 1986, 212); and this, too, is apparently maintained by Varzi (2001, 146-148). Moreover, precisely this denial of de re vagueness works 
as a key assumption in Lewis's argument for Unrestricted Composition in On the Plurality of Worlds. ${ }^{2}$

Let us stick, then, to Assumptions (1) and (2): there is such a thing as identity simpliciter, and it admits no de re indeterminacy. Next, let us assume as our sample world a "restricted model": there are just two non-overlapping objects Out There, $x$ and $y$. Let us also straightforwardly assume that they have no proper parts (we may also take them as having proper parts, but this would just complicate the calculation without changing the point of the argument). The Minimalist View tells us that, in order to "weigh our ontological commitments", we should count either the parts, or the whole. We can do exactly one of two things: we can count as items in our "inventory of the world" $x$ and $y$; or, we can count only $z$ (that is, $x+y$ ); for the formers overlap the latter and, according to Principle (M), mentioned in Varzi's quotation above, we should not include overlapping entities.

The Minimalist View does not tell us which option(s) should be favored: "one mereologist may go for a fine-grained inventory including $x$ and $y$ but not $x+y$; another mereologist may go for a coarser inventory including $x+y$ but not $x$ or $y$ " (Varzi 2000,287): $x$ and $y$ may be too disconnected and independent to count $x+y$ as a full-fledged object (in this case, calling $x$ and $y$ "parts" may sound odd to begin with - parts of what?). Or the $z=x+y$ may be too self-connected to count $x$ and $y$ as full-fledged objects (in this case, calling the one relevant object around, namely, $z, " x+y$ " may sound odd to begin with - a fusion of what?).

But whatever we choose, neither one nor two is three. And so the Minimalist View is incompatible with mereology. The latter asserts the existence of three objects, which the former denies. To be "included in the inventory of the world" a general catalogue of all that is Out There - is nothing more and nothing less than to exist. But then, to claim that, if one has already "included in the inventory of the world" $x$ and $y$, one should not include $x+y$, and vice versa, is to claim that if one is committed to the existence of $x$ and of $y$, one should not concede an existence ticket also to $x+y-$ and vice versa. But this is precisely what mereology does.

\section{Against innocent mereology and double existence}

The Minimalist View avoids one problem of mereology at the cost of getting a new one. The problem it avoids concerns the ontological innocence of mereology. The cost is the introduction of a double notion of existence. Let us see how.

2 The argument, roughly, goes as follows: our restrictions on composition depend on vague intuitions and admit of degrees. But the issue whether, given that $x$ and $y$ are Out There, their mereological sum $z=x+y$ is Out There, too, can be formulated in a (fragment of) language in which nothing is vague. Therefore (given that there is no de re vagueness), it cannot have a vague answer: either there is such a thing as $x+y$, or there isn't. So "no restriction on composition can be vague. But unless it's vague, it cannot fit the intuitive desiderata" (Lewis 1986, 213). Consequently, any restriction on composition would be unmotivated. 
If it is true that mereology is ontologically innocent, as Lewis has argued, then once we are committed to the existence of $x$ and $y$, our commitment to $z$, i.e., $x+y$, is, in a sense, not a further commitment. In Lewis's own words:

Given a prior commitment to cats, say, a commitment to cat-sums is not a further commitment. The sum is nothing over and above the cats that compose it. It just is them. They just are it. Take them together or take them separately, the cats are the same portion of Reality either way. Commit yourself to their existence all together or one at a time, it is the same commitment either way ... I say that composition . . . is like identity. The 'are' of composition is, so to speak, the plural form of the 'is' of identity. Call this the Thesis of Composition as Identity. It is in virtue of this thesis that mereology is ontologically innocent: it commits us only to things that are identical, so to speak, to what we were committed to before (Lewis 1991, 81-82).

At least prima facie, the view of composition as identity could make mereology's unrestricted composition more palatable, by advocating its ontological innocence. Lewis's argument for the innocence of mereology can be summarized in the following way:

(P1) Composition - a many-one mereological relation - is like identity.

(P2) Nothing could be considered more ontologically innocent than the request to accept something identical to things already accepted.

(C) Therefore, mereology is ontologically innocent.

For Lewis, the sum $z$ of certain objects $x$ and $y$ is the very same objects: the sum $z$ is those things, $x$ and $y$, and nothing more.

Lewis's argument rests on the thesis (P1) of composition as identity. ${ }^{3}$ What does the claim that composition is like identity mean? The answer depends on the reading of $(\mathrm{P} 1)$ one accepts. In fact, there are two of them: a strong reading (StrongCom) and a weak one (WeakCom). The former goes as follows:

(StrongCom) The predicate "are" used for the composition relation is literally the plural form of the "is" of identity.

Formally:

$\forall X \forall z((z$ is the sum of the $X) \rightarrow z=X)$,

${ }^{3}$ The view of composition as identity has been recently discussed in detail in Ted Sider's paper Parthood. For Sider, "Composition as identity is intuitively appealing. It is a little wild in its strong form, though not as indefensible as it first seems. Still, it should be rejected, since it undermines the theoretical utility of irreducibly plural quantification. A tamer moderate form stays as faithful as possible to the motivating idea: that a composite is nothing over and above its parts, that things just are their parts. And it flows from a more general conception of parthood as a uniquely intimate relation. That conception explains why intrinsic properties of parts give rise to intrinsic properties of wholes, and why things are located where their parts are. It supplies a powerful argument for classical mereology, and an argument against coincident entities and most forms of endurance" (Sider 2007, 88). 
where ' $X$ ' stays for a plural variable (i.e. a variable that can refer to pluralities). Those who accept (StrongCom) argue that the sum $z$ of some things is literally identical to those things: the things are their sum; the sum is those things.

In the second reading of (P1) - the weak reading of composition, (WeakCom) - the composition predicate is only analogous to identity. (WeakCom) is formulated in the following way:

(WeakCom) The predicate "are" used for the composition relation is analogous to the plural form of the "is" of identity.

The strength of Lewis's argument for the innocence of mereology depends on the truth of (P1), i.e. on the truth either of (StrongCom) or of (WeakCom). Unfortunately, neither (StrongCom) nor (WeakCom) are true. Lewis himself formulates two arguments against (StrongCom) in Lewis $(1991,87)$. So we do not take care about it here. Instead, he argues for (WeakCom): the relation between composition and identity is analogical. In this second reading of (P1) one is confined to argue for some similarities between composition and identity: however, the analogies are claimed to be such that it is appropriate to say that composition "is, so to speak, the plural form" of identity. Lewis lists the following aspects of similarity:

(Unrestricted composition) Just as everything is identical to something, likewise, given anyway some $X$, they compose something. So, for example, there is no special condition Argle must satisfy to be identical to himself. Likewise, there is no special condition Argle and Bargle must satisfy to compose something.

(Uniqueness of Composition) Just as there cannot be anything identical with two distinct objects, likewise there cannot be two distinct sums of the same objects. For example, there cannot be two distinct things both identical to Argle. Likewise, there cannot be two objects both composed exactly by Argle and Bargle.

(Ease of Describing Sums) Just as if you fully describe the thing $x$ you fully describe something identical to $x$, likewise if you fully describe the $X$ you fully describe their sum. For example, you can fully describe the object identical to Argle by describing Argle. Likewise, you can fully describe the object, Arglebargle, composed by Argle and Bargle, by fully describing Argle and Bargle.

(The spatial coincidence) Just as $x$ and $y$ have to occupy the same spatiotemporal region if the first object is identical to the second, likewise $y$ and $X$ have to occupy the same spatiotemporal region if $y$ is the sum of the $X$. For example, if there is an object Argle in a certain place at a certain time, Argle exists in the same place and time. Likewise, Arglebargle is in the same spatiotemporal region occupied by Bargle and occupied by Argle.

For Lewis, since composition and identity are analogous in these respects, it is appropriate to expand the meaning of "identity" to cover both composition and identity: what we used to call identity is a special limiting case of what is now called "identity". 
On the basis of the following analogies, a defence of the ontological innocence of mereology is proposed. In fact, from:

(1) There is a certain object $x$, a dog, which is sleeping,

and

(2) There is a certain object $y$, a cat, which is eating,

it follows that:

(1*) There is something the object $x$, a dog, is;

$(2 *) \quad$ There is something the object $y$, a cat, is.

For Lewis, from $\left(1^{*}\right)$ and $\left(2^{*}\right)$, just considering the "are" of composition as, so to speak, a plural form of the "is" of identity, it follows that:

(3*) There is something the objects $x$, a dog, and $y$, a cat, are,

where the "something" in $\left(3^{*}\right)$ is - for Lewis - the sum, $z$.

There is - for Lewis - no further commitment in $\left(3^{*}\right)$, after the commitment of $\left(1^{*}\right)$ and $\left(2^{*}\right)$, just as there is no further commitment in $\left(1^{*}\right)$ after the commitment in the assertion of (1), and ditto for ( $\left.2^{*}\right)$ and (2). "The gist of the argument is this: because the composition predicate in sentence $\left(3^{*}\right)$ is analogous to the identity predicate in sentence $\left(2^{*}\right)$ in the four respects mentioned above, it is analogous in yet another respect in question" (Yi 1999, 152-153), i.e. just as (3*) is redundant given $\left(1^{*}\right)$ and $\left(2^{*}\right)$, it is just a logical consequence of the two sentences, so $\left(2^{*}\right)$ is redundant given the prior assertion of the existence of a cat in (2) because it is just a logical consequence of the previous sentence. ${ }^{4}$

We think that Lewis's argument is wrong. First of all, the sentence ( $\left.3^{*}\right)$ does not seem to be a consequence of $\left(1^{*}\right)$ and $\left(2^{*}\right)$. What follows from $\left(1^{*}\right)$ and $\left(2^{*}\right)$ is:

(3**) There are the object $x$, a dog, and the object $y$, a cat.

But ( $3 * *)$ simply says that they both exist. In fact, we can paraphrase $(3 * *)$ in the following way:

$(3 * * *)$ There is something the object $x$, a dog, is, and there is something the object $y$, a cat, is.

The problem is that $(3 * * *)$ does not entail $(3 *)$. Formally, from:

${ }^{4}$ One referee has suggested that one could argue that, though WeakCom does not entail the commitment to sums, it helps to explain the commitment to sums. In fact, Lewis doesn't talk about logical consequence. But, then how to understand the fact that $\left(3^{*}\right)$ follows from $\left(1^{*}\right)$ and $(2 *)$ ? Logical consequence, nothing more than that, seems to be here at work. 


$$
\exists x(x=a) \wedge \exists x(x=b)
$$

it does not follow that:

$$
\exists x(x=a \wedge x=b) .
$$

It seems to us that those who object that (5) is not the correct paraphrase of $\left(3^{*}\right)$ - because in $\left(3^{*}\right)$ one says that there is something $x$ and $y$ are collectively, not distributively - are wrong. In fact, the conjunction of (1) and (2) says that $x$ and $y$ are each of them something. From that by no means follows that they are something collectively.

The second problem of Lewis's argument for (WeakCom) concerns some specific aspects of the analogy. Consider, for example, the Ease of Describing Sums. According to this analogy, just as if you fully describe the thing $x$ you fully describe something identical to $x$, likewise if you fully describe the $X$ you fully describe their sum. In fact the analogy seems to be wrong. Certainly, a full description of the thing $x$, say, $D(x)$, will be a full description, $D(y)$, of a $y$ such that $y=x$. However, given certain $X$ and $Y$, the sum of the $X$ could be identical to the sum of the $Y$ even if the $X$ are not identical to the $Y$. Now, if the $X$ are not identical to the $Y$, then a full description of the $X, D(X)$, is quite likely to be different from a full description of the $Y, D(Y)$. So these two different descriptions, $D(X)$ and $D(Y)$, cannot both be full descriptions of the unique thing which is the sum of the $X$, and also the sum of the $Y$. Therefore, at least one full description of the things that compose a sum will not be a full description of the sum itself.

Here is an example. Consider a rectangle A:

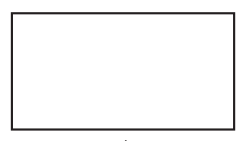

A

The rectangle A can be composed as the mereological sum of two squares, or of two triangles.

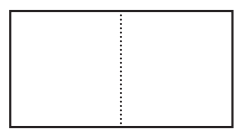

A

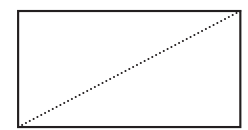

A

However, a full description of the squares, $D$ (Squares), will differ in content from a full description of the triangles, $D$ (Triangles) (after all, the squares are squares, which the triangles are not; so the former will include something like "those items are squares", whereas the latter will not). Now take the full descrip- 
tion of the rectangle A. If both $D$ (Squares) and $D$ (Triangles) were full descriptions of the unique thing which is the sum of the squares and also the sum of the triangles, namely of the rectangle $\mathrm{A}$, we would have that two full descriptions with different contents, $D$ (Squares) and $D$ (Triangles), are full descriptions of the very same thing, namely A, which is absurd.

Assume, then, that mereology is not innocent in the Lewisian sense, and let us go back to the Minimalist View. Could a strategy like the Minimalist View, which was supposed to help mereology with its prima facie implausibility and to resolve the "tension between countenancing and counting", lead to a quick, obvious, and straightforward solution of the issue of the ontological innocence of mereology? Things may not be so clear-cut, because the Minimalist View also calls for a duplication of the senses in which something can be in an "inventory of the world" - of the senses in which something can be Out There as an object in the domain of quantification. It calls, in other words, for a duplication of the notion of existence. Here is how the duplication is explicitly introduced in Varzi's paper:

The Minimalist View draws a distinction between two senses in which a thing can be object of reference or quantification - two notions of individual existence. In one sense, those items exist that are included in the domain of quantification of our part-whole theory, whatever it is (call this the Quinean notion of existence). [. . .] On the other hand, there is a sense in which just those items exist that are listed in the relevant inventory of the world depending on how this is drawn up. These items will all be included among those things that enjoy Quinean existence, since they will have to be recognized as objects of reference and quantification by the part-whole theory: but not everything that exists in the Quinean sense must exist in this restricted sense (the selective notion of existence) (Varzi 2000, 286-7).

However, someone may find this duplication between "Quinean existence" and "selective existence" hardly justifiable. It may be difficult, in other words, to understand what a difference in senses of "existence" might be.

Some philosophers have been tempted by the idea that "there is", or "exists", can have different meanings when ascribed to different kinds of things, such as abstract objects, material ones, or minds (see, famously, Ryle 1949). But the following simple remark (due to van Inwagen 1998) speaks strongly on behalf of the fact that existence is univocal. To say that there are cats is to say that the number of cats is one or more; and to say that there are no unicorns is to say that the number of unicorns is zero. In a motto: to exist is to count (or, better: to count as one). ${ }^{5}$ Now, number words are definitely univocal: it cannot be the case that the number of the Apostles and the number of months in a year are not the same

${ }^{5}$ Entities denoted by mass terms like "water" and "gold" are sometimes considered exceptions to our motto. Consider for example a sentence like "water has been pouring into the basement all night". The sentence might be true, and if so, presumably, there must be something to which the mass term "water" refers. But it is not one, and it is not many. On this topic see (Laycock 2006) (Thanks to one of the referees for the suggestion). 
number, given that the number of the Apostles is 12 and the number of months in a year is also 12. It will not do, now, to resort to the profound ontological differences between persons and days of the week and claim that " 12 " should not be understood in the same sense in both cases. The number of the Apostles is the number of months in a year. Some things can exist on earth, and some in heaven; some, like numbers themselves, according to many exist in no place at all. However, no matter how much these various objects may differ, their differences make no difference in their existence.

We are here adopting Frege's theory of counting. Frege's doctrine figures as the conclusion of an argument that starts at section 22 of the Grundlagen. The argument aims at showing that the real bearers of numbers are concepts and not ordinary objects (or ordinary external events) or collections, as a naive theory of numbers would have it. Frege's strategy is to prove that thesis by a reductio ad absurdum of the opposed thesis taken as a hypothesis. It runs roughly as follows. If the real bearers of numbers were ordinary objects or ordinary external events or collections then there would be no absolute sense in which a given number rather than another could be said to belong to its bearer. The reason is that a given object or a collection can be thought of in many different ways. Take for example the Iliad. One could think of it as one poem, as twenty-four books, or as a large number of verses. Or take a group of soldiers: one could think of it as one army, two divisions, or five hundred men. The ascription of a number to something would therefore be relative. But what would it be relative to? According to Frege, in order to provide an answer to that question one has to consider what changes in the passage from one expression to another. And here is what he says:

Now, what changes here from one judgment to the other is neither any individual object nor the whole, the agglomeration of them, but rather my terminology. But that is itself only a sign that one concept has been substituted for another (Frege 1884, section 46,59$)$.

For Frege, then, any ascription of a number to something is always relative to a concept, introduced by a general term (or as he says: "The content of a statement of number is an assertion about a concept"). The concept role is to make counting possible by specifying, in each case, the nature of the task to perform, or - as Frege puts it - the object of the investigation. For example, if we say:

Venus has zero moons,

we are ascribing a certain property to the concept Moon of Venus, i.e. the property of having an empty extension. Instead, if we say:

Jupiter has four moons,

we are saying that the number which belongs to the concept Moon of Jupiter is four, or that the concept has four unities in its extension. Thus, when I say, pointing to a pile of cards: 
(8) The number of these is two

I might have in mind either the sentence:

(9) The number two belongs to the concept pack contained in this pile

or the sentence:

(10) The number two belongs to the concept cards contained in this pile.

Following Frege, counting things under more than one concept at once might result in mistakenly counting things twice. So, supposing we want to buy a pack of six colas. We might wonder if our six pack counts as one thing, six things, or seven. For sure, we cannot count six colas and one pack of cola. Things are not counted simpliciter; they are counted under a certain sortal. Under one sortal they can be many, under another sortal something can be one.

The fact Frege's conception of counting prescribes to count things under a concept does not mean it violates our Assumption (1) - shared by Lewis and Varzi - that is, that there is such a thing as identity simpliciter. Frege's doctrine of cardinality implies no relativization of identity. Suppose, for example, a rancher in the habit of naming all his herds and cows. He points in the direction of the same cow but of different herds, on successive days (supposing that the cow has changed pastures on the successive days). He says:

(11) That is one

(11) may be ambiguous. We may mean one heard or one cow. But, once it is clear which (kind of) entities are in question, there is an immediate connection with absolute identity:

If there is one herd then, for any herd $x$ and $y, x=y$,

If there is one cow then, for any cow $p$ and $q, p=q$.

What is important to notice is that once the (kind of) entities in question are chosen there is nothing left to be specified: we do not have to choose between a variety of identity-like relations.

If we take seriously Frege's doctrine of counting we obtain the justification of our motto: to exist is to count. Take (11) again: from a Fregean point of view what is said in (11) or in sentences of the form:

$$
x \text { is one }
$$

is that the concept being identical with $x$ has the property of having a singular exemplification, or, in other terms, that there is something identical with $x$.

Once adopted Frege's conception of counting let us consider again the Minimalist view. Varzi goes on claiming: 
So this is how the Minimalist View takes the fundamental tension of mereology to be explained. We quantify over everything, since the meaning of 'everything' is set by the domain of the quantifiers; yet counting is selective (Varzi 2000, 287).

Our problem with the maxim "We quantify over everything [...] yet counting is selective" should be clear by now. We have a problem with its (ambiguously) parting quantifying and counting, if both are given strict metaphysical import. One cannot part quantifying and counting, i.e. existence and numbers: to be is to be the value of a variable, that is, to be is to be in the domain of quantification, and to be is to exist, and to exist is to count as one.

But if this is so, how can we make sense of the distinction between "Quinean existence" and "selective existence"? One cannot claim that "three" has different meanings when she "speaks mereologically" (and, referring to our restricted universe above, she claims that there are three objects), and when she "speaks Minimalistically" (and she denies that there are three objects). The mereological principle of Unrestricted Composition tells us that, given $x$ and $y$, there exists a $z$ such that $z=x+y$. And if existence is univocal, we are committed to the existence of $x, y$ and $z$. Now the Minimalist View tells us that, if we have already counted $x$ and $y$ in our inventory of the world, we should not count $z$ (that is, $x+y$ ); and if we have already counted $z$ (that is, $x+y$ ) in our inventory of the world, we should not count $x$ or $y$. To us, this can only mean that if there are such things as $x$ and $y$, then there is no such thing as $z$; and if there is such thing as $z$, then there are no such things as $x$ or $y$. In both cases, the things that should exist in order for mereology to be true of the world are not Out There.

A good inventory - one that reflects our ontological commitments - is not simply a list of all the pieces that we have got, a list of all that can enter our parthood relation. On the Minimalist View, a good inventory is more selective: it must meet the constraint in (M) (Varzi 2000, 286).

But mereology aims at providing, via its theorems, precisely "a list of all that can enter our parthood relation". So if a good inventory of the furniture of the world that is, one produced with the help of the Minimalist View - is "more selective" (i.e. it leads us to count as objects less items than those provided by "a list of all that can enter our parthood relation"), this can only mean that mereology is not a good inventory of all there is Out There. We may have, at the same time, two different "inventories of the world", but if they differ in the number of items they include, and if it makes sense to speak simpliciter of the number of objects Out There, given that existence is univocal, then one of them must be wrong.

The fact that the Minimalist View does not force upon us a single inventory, (e.g. because it allows us the option of countenancing $x$ and $y$ in it, or alternatively the option of countenancing $x+y$ ) is irrelevant; for none of the allowed inventories will be compatible with mereology itself, which has in its own inventory $x, y$, and 
$z$ (i.e., $x+y$ ). Mereology may be a "basis" for all the inventories one can write down with the help of the Minimalist View; but only in order to be refuted by each one of them.

\section{Things that count: the conceptual way out}

We claim that the only way for the mereologist to make the Minimalist View work on behalf of mereology is to disambiguate the Minimalist View itself into what we will call an epistemic or conceptual interpretation - by which we mean what follows: we should not say anymore that the Minimalist View distinguishes between a "primordial, Quinean notion of existence (to be is to be included in the domain of our mereological theory)", and a "more selective, cognitively relevant notion (to be is to be included in an inventory of the world, however this is drawn up)" (Varzi 2000, 300). The proposed disambiguation requires the mereologist to admit that "primordial, Quinean existence" is nothing but existence. And there is no room for a second, "selective, cognitively relevant existence". We had better claim that the Minimalist View accounts for how we select as cognitively salient some of the things that exist. This could allow us to avoid problems of implausible ontological commitments without a duplication of existence.

Let us have a more detailed look at the strategy, following Frege's insights. First, an ontologist who subscribes to classical mereology can have her cake and eat it by admitting that "Quinean existence" is the only sense of existence available; and that all and only those things exist (in the only available sense of "exists"), that are included in the domain of quantification of classical mereology. ${ }^{6}$ In particular, given that $x$ and $y$ are Out There, also $z=x+y$ should be Out There - it should be countenanced in the "inventory of the world".

Second, our common counting procedures are nevertheless sortalized (say, a pack of of six colas is counted as six colas and one pack of cola, for instance). The principle (M) of the Minimalist View prescribes that, after listing a table as an item in our inventory, we should not countenance its parts too-say, the legs and the top. Appropriately disambiguated, this cannot mean anymore that table, legs and top all exist in one sense, whereas only the table exists in another sense. For there aren't two senses of existence; the disambiguated claim is that table, legs, and top all exist, full stop, but to count them we need to sortalize them under different sortal concepts and, as soon as we adopt a sortal, counting starts.

Once such a conceptual disambiguation is given, the Minimalist View may become compatible with classical mereology. Mereology, the mereologist is now

${ }^{6}$ As one anonymous referee has suggested to us, this issue also turns on the collateral problem of whether mereological sums have their parts essentially. In this paper we would like to be neutral about the status of mereological sums: so, as the generality of the theory does not permit to exclude unsought sums for reasons concerning the nature or location of the taken objects, likewise it does not permit to include just sums with essential parts. 
allowed to claim, tells us what is Out There (what does exist, in the only available sense of the word). You "speak mereologically" when you speak with your quantifiers wide open, and this appears to commit you to strange entities of various kinds (and sometimes, to entities that seem to belong to no kind at all).

On the other hand, the Minimalist View can account for the conceptual procedures that lead us to select, in a wide range of cases, parts at the expense of wholes, or vice versa. So disambiguated, it can provide an interesting explanation of our counting policies. For instance, the principle of maximal self-connectedness of an object advanced by Varzi seems to point exactly towards an "epistemic" and contextual interpretation: it is relativized to kinds, "properties or conditions of qualitative homogeneity"; and "what these conditions are may be matter of controversy and may depend heavily on context and cognitive factors" (Varzi 2000, 299).

The Minimalist View does not even force upon us a unique counting policy, for it acknowledges that, sometimes, we may want to count undetached parts separately (say, bananas in a bunch), and sometimes we may want to count disconnected wholes as one (say, bikinis). It helps us to understand that mereology seems implausible because we have forgotten (or, most often, we simply have never paid attention to) objects in which we have no practical interest, for which we have no names, or no perceptual sympathy. This will not make them go away metaphysically, though (on this point, see also van Cleve 1986, 145). The counting and quantifying policies the Minimalist View accounts for are not policies for establishing what is Out There: they are different ways to focus our cognitive attention to some of the things that were, are, and will keep staying, Out There.

However, even if the cognitive or conceptual reading of the Minimalist View could make it compatible with mereology, it would no more help Varzi's original purposes of resolving the tension in mereology than does a metaphysical reading on multiplying existence. Mereology says that there is an object composed of, say, the middle third of a baseball bat and the southern half of the Empire State Building; common sense says that there is no such object. And if the common sense view provides a genuine alternative ontology to mereology, as some have argued (see e.g. van Inwagen 1981), then even the epistemic reading of Varzi's Minimalist View would be no help to resolving the tension between mereology and common sense. ${ }^{7}$

${ }^{7}$ Besides, the Minimalist View is clearly a prescriptive view: it says what the ontologist should and should not count in her inventory of the world (and the maxim (M) above is clearly prescriptive). However, in its conceptual reading it is a mere account of the cognitive procedures which leads us to select parts at the expense of wholes or vice versa, and therefore, it is merely descriptive. Thanks to an anonymous referee for pointing this out to us. 


\section{REFERENCES}

Chisholm, R.M. 1987, 'Scattered Objects', in: J.J. Thomson, ed., On Being and Saying, Cambridge MA: MIT Press, pp. 167-173.

Cleve, J. van 1986, 'Mereological Essentialism, Mereological Conjunctivism, and Identity Through Time', Midwest Studies in Philosophy 11, pp. 141-156.

Frege, G. 1884, Die Grundlagen der Arithmetik. Eine logisch-matematische Untersuchung Über den Begriff der Zahl, Breslavia: Kàbner. Trans by J. L. Austin, The Foundations of Arithmetic, Oxford: Basil Blackwell, 1968.

Inwagen, P. van 1981, 'The Doctrine of Arbitrary Undetached Parts', Pacific Philosophical Quarterly 62, pp. 123-137.

InWagen, P. van 1998, 'Meta-ontology', Erkenntnis 48, pp. 233-250.

Inwagen, P. van 2002, 'The Number of Things', Philosophical Issues 12, pp. 176-196.

LAYCOCK, H. 2006, Words without Objects, Oxford: Oxford University Press.

LewIs, D. 1986, On the Plurality of Worlds, Oxford: Blackwell.

LewIs, D. 1991, Parts of Classes, Oxford: Blackwell.

RYLE, G. 1949, The Concept of Mind, London: Hutchinson.

Sider, T. 2007, 'Parthood', The Philosophical Review 116, pp. 51-91.

VARZI, A. 2000, 'Mereological Commitments', dialectica 54, pp. 283-305.

VARZI, A. 2001, Parole, oggetti, eventi e altri argomenti di metafisica, Roma: Carocci Editore.

YI, B. 1999, 'Is Mereology Ontologically Innocent?' Philosophical Studies 93, pp. 141-160. 